Review of Income and Wealth

Series 57, Number 1, March 2011

\title{
UPWARD STRUCTURAL MOBILITY, EXCHANGE MOBILITY, AND SUBGROUP CONSISTENT MOBILITY MEASUREMENT: U.S.-GERMAN MOBILITY RANKINGS REVISITED*
}

\author{
By Christian Schluter
}

University of Southampton

AND

DIRK VAN DE GAER*

SHERPPA, Ghent University and CORE, Université Catholique de Louvain

\begin{abstract}
We formalize the concept of upward structural mobility and use the framework of subgroup consistent mobility measurement to derive a relative and an absolute measure of mobility that is increasing in upward structural mobility and compatible with the notion of exchange mobility. In our empirical illustration, we contribute substantively to the ongoing debate about mobility rankings between the U.S. and Germany by demonstrating that the U.S. typically does exhibit more upward structural mobility than Germany.
\end{abstract}

\section{INTRODUCTION}

Exchange and structural mobility are old and recurrent concerns in economics and sociology. Informally, exchange mobility is said to increase if the correlation between incomes in two successive periods decreases while keeping the marginal income distributions constant. By contrast, structural mobility compares situations that have different marginal distributions in the two periods. It amounts to factual mobility "caused by differential change in the stratum distribution" (Yasuda, 1964, p. 16) or-in an intergenerational context-by "the amount of mobility generated by the fact that the distribution among social strata experienced by the sons differs from the corresponding experience of their fathers" (Boudon, 1973, p. 17). The formal literature has focused on exchange mobility, whereas the concept of upward structural mobility has received only little attention.

However, students' verbal responses in questionnaire studies generally agree that structural mobility matters in both the measurement and evaluation of mobility (Bernasconi and Dardanoni, 2005). Moreover, to the best of our knowledge,

Note: We thank Thomas Demuynck, Udo Ebert, Patrick Moyes, two anonymous referees, and the Co-Managing Editor for detailed and constructive comments. We also thank the participants of the IRISS-C/I 10th Anniversary Workshop (Differdange, October 24-25, 2008) and the Conference on Income and Earnings Dynamics (Maynooth, July 3, 2009). We are grateful to our referees for their constructive comments which have led to an improved paper. This paper presents research results of the Belgian Program on Interuniversity Poles of Attraction initiated by the Belgian State, Prime Minister's Office, Science Policy Programming. The scientific responsibility is assumed by the authors.

*Correspondence to: Dirk Van de gaer, SHERPPA, Vakgroep sociale economie, F.E.B., Ghent University, Tweekerkenstraat 2, B-9000 Gent, Belgium (Dirk.Vandegaer@ugent.be).

(C) 2010 The Authors

Review of Income and Wealth (C) 2010 International Association for Research in Income and Wealth Published by Blackwell Publishing, 9600 Garsington Road, Oxford OX4 2DQ, UK and 350 Main St, Malden, MA, 02148, USA. 
upward structural mobility has never been analyzed in the axiomatic literature. We provide a formal definition of the concept such that more upward (downward) structural mobility increases (decreases) the index and show that it can be reconciled with exchange mobility. It should be remembered, however, as stressed in Fields (2007), that mobility is a multifaceted concept and other notions of structural mobility, such as undirected ones, are conceivable.

We conduct our analysis within the framework of replication invariant, subgroup consistent mobility measurement. This class has a strong intuitive appeal as the following motivating example illustrates. Suppose we manage to identify a group in the population with a low income mobility (e.g. in an intergenerational context children from blue collar workers). If we manage to increase this group's upward mobility while keeping the mobility of other groups constant, then subgroup consistency ensures that total mobility in society has increased. ${ }^{1}$ Subgroup consistency has long been accepted in inequality and poverty analyses as an important property; it is only recently that researchers have considered and formulated subgroup consistent mobility measures. Moreover, Schluter and Trede (2003) have highlighted the importance of transparent aggregation of income changes, and subgroup consistency ensures such transparency. We derive axiomatically simple replication invariant subgroup consistent relative and absolute mobility indices that are increasing in exchange mobility and increasing (decreasing) in more upward (downward) structural mobility.

We demonstrate the usefulness of our framework by revisiting the ongoing debate about income mobility comparisons between the U.S. and Germany (see, e.g., Burkhauser and Poupore, 1997; Burkhauser et al., 1997; Maasoumi and Trede, 2001; Gottschalk and Spolaore, 2002; Schluter and Trede, 2003). The point of departure of this debate is the observation that when using standard mobility measures Germany is ranked, contrary to received wisdom, more mobile than the U.S. We contribute substantively to this debate by (i) showing that the standard mobility measures are inconsistent with our notions of upward structural and exchange mobility, and (ii) demonstrating that the U.S. typically does exhibit more upward structural mobility than Germany.

The outline of this paper is as follows. In the next section we introduce the framework and core axioms, and axiomatize the two notions of mobility. In Section 3 we derive a class of mobility measures which are increasing in upward structural mobility and exchange mobility. Section 4 presents our empirical illustration about mobility rankings between the U.S. and Germany. All proofs are gathered in the Appendix.

\section{Notation And Axioms}

Let $y_{1} \in \mathbb{R}_{++}^{n}$ be transformed through some dynamic process into $y_{2} \in \mathbb{R}_{++}^{n}$; the vector $\left(y_{1}, y_{2}\right)$ belongs to $D=\bigcup_{n=1}^{\infty} \mathbb{R}_{++}^{2 n}$. In this paper we examine subgroup consistent mobility measures. To this end index the group by $g$, and, for notational

\footnotetext{
${ }^{1}$ Total mobility increases irrespective of how the change affects the relative positions of the remaining population. Foster and Sen (1997, pp. 156-63) discuss the pros and cons of this argument in the context of inequality measurement.
} 
simplicity, consider only two groups of individuals with $g \in\{1,2\}$. Let $P=\left\{N^{1}\right.$, $\left.N^{2}\right\}$ be a partition of the set $N=\{1, \ldots, n\}$ in two non-overlapping subsets and let $\mathcal{P}$ denote the set of all such possible partitions of $N$. For each group $g$ of size $N^{g}$, the income vectors are partitioned correspondingly into $\left(y_{1}^{g}, y_{2}^{g}\right)$.

A replication invariant subgroup consistent (RISC) mobility index is a nonconstant function $M: D \rightarrow \mathbb{R}$, continuous in its arguments, whose value indicates the amount of mobility in moving from a distribution $y_{1}$ to $y_{2}$, and which satisfies the following axioms:

RISC. 1 [Anonymity]: $M\left(y_{1}, y_{2}\right)$ is symmetric: $M\left(y_{1}, y_{2}\right)=M\left(y_{1}^{\prime}, y_{2}^{\prime}\right)$ whenever the vectors $y_{1}^{\prime}$ and $y_{2}^{\prime}$ are obtained after applying the same permutation on $y_{1}$ and $y_{2}$.

RISC.2 [Replication Invariance]: $M\left(y_{1}, y_{2}\right)=M\left(y_{1}^{\prime}, y_{2}^{\prime}\right)$, whenever the vectors $y_{1}^{\prime}$ and $y_{2}^{\prime}$ are obtained after applying the same replication on $y_{1}$ and $y_{2}$.

RISC.3 [Subgroup Consistency]: For all $P \in \mathcal{P}: M\left(y_{1}^{1}, y_{1}^{2}, y_{2}^{1}, y_{2}^{2}\right)>$ $M\left(y_{1}^{1 \prime}, y_{1}^{2 \prime}, y_{2}^{1 \prime}, y_{2}^{2 \prime}\right)$ whenever $M\left(y_{1}^{1}, y_{2}^{1}\right)>M\left(y_{1}^{1 \prime}, y_{2}^{1 \prime}\right)$ and $M\left(y_{1}^{2}, y_{2}^{2}\right)=M\left(y_{1}^{2 \prime}, y_{2}^{2 \prime}\right)$.

Anonymity is generally accepted as a property for mobility measures. Replication invariance is also very common. The third requirement is subgroup consistency which has been considered in the literature on mobility measurement before (see, e.g., Fields and Ok, 1999; D’Agostino and Dardanoni, 2009).

We now formalize our two core mobility properties. Our first mobility axiom concerns exchange mobility. Exchange mobility is a property of the joint distribution of incomes in both periods while the marginal distributions in both periods are kept fixed. It requires that if there is a positive association between the incomes in two periods for two pairs of incomes $\left(y_{1 i}, y_{2 i}\right)$ and $\left(y_{1 j}, y_{2 j}\right)$, then swapping $y_{2 i}$ and $y_{2 j}$ or swapping $y_{1 i}$ and $y_{1 j}$ increases mobility since it decreases the positive association between the income vectors of both periods. Formally, given a vector $x=\left(x_{1}, \ldots, x_{i}, \ldots, x_{j}, \ldots, x_{n}\right) \in \mathbb{R}_{++}^{n}$, define $x\left(\sigma_{i j}\right)=\left(x_{1}, \ldots, x_{j}, \ldots, x_{i}, \ldots, x_{n}\right) \in \mathbb{R}_{++}^{n}$. The Exchange Mobility (EM) axiom states:

EM: For all $i, \quad j \in N \quad$ and all $\quad y_{1}, y_{2}, y_{1}\left(\sigma_{i j}\right), y_{2}\left(\sigma_{i j}\right) \in \mathbb{R}_{++}^{n}$ :
$\left(y_{1 i}-y_{1 j}\right)\left(y_{2 i}-y_{2 j}\right)>0 \Rightarrow M\left(y_{1}, y_{2}\left(\sigma_{i j}\right)\right)>M\left(y_{1}, y_{2}\right)$ and $M\left(y_{1}\left(\sigma_{i j}\right), y_{2}\right)>M\left(y_{1}, y_{2}\right)$.

While the interpretation of $\mathrm{EM}^{2}$ is clear and EM is often discussed in the literature (see, e.g., Atkinson, 1981; Markandya, 1982; Dardanoni, 1993) its implications for a mobility index of the general form $M\left(y_{1}, y_{2}\right)$ are not. By imposing more structure on the mobility index using the RISC axioms we uncover the implications of EM for RISC mobility measures in Section 3.

Our next set of axioms concerns the notion of structural mobility. We formalize the core concept of upward structural mobility first before considering additional notions of structural mobility. Upward structural mobility is concerned with changes in the marginal distributions. It consists of two

\footnotetext{
${ }^{2}$ Both swaps in the EM axiom decrease the joint probability distribution at $\left(y_{1 i}, y_{2 i}\right)$ and $\left(y_{1 j}, y_{2 j}\right)$ and increase it at $\left(y_{1 i}, y_{2 j}\right)$ and $\left(y_{1 j}, y_{2 i}\right)$. Thus, since the condition on the swaps is that $\left(y_{1 i}-y_{1 j}\right)\left(y_{2 i}-y_{2 j}\right)>0$, probability mass is pushed away from the diagonal. This is the equivalent, in the discrete case, of the mobility increasing transformation as introduced by Atkinson (1981).
} 
components. Given a vector $x=\left(x_{1}, \ldots, x_{i}, \ldots, x_{n}\right) \in \mathbb{R}_{++}^{n}$, consider any $\varepsilon \in \mathbb{R}_{++}$ such that $x^{+\varepsilon(i)}=\left(x_{1}, \ldots, x_{i}+\varepsilon, \ldots x_{n}\right) \in \mathbb{R}_{++}^{n}$ Clearly the distribution $x^{+\varepsilon(i)}$ first order stochastically dominates the distribution $x$. (i) Assume that starting from $y_{1}$ a second period income distribution $y_{2}^{+\varepsilon(i)}$ is reached instead of $y_{2}$. The process that changes $y_{1}$ into $y_{2}^{+\varepsilon(i)}$ has more upward structural mobility than the process changing $y_{1}$ into $y_{2}$ since, starting from the same initial distribution of incomes (or social strata), a better distribution of income (with more desirable social strata) is reached. (ii) Assume now that a second period income distribution $y_{2}$ is reached from a first period income distribution $y_{1}^{+\varepsilon(i)}$ instead of from $y_{1}$. Here, the process that changes $y_{1}^{+\varepsilon(i)}$ into $y_{2}$ has less upward structural mobility than the process that changes $y_{1}$ into $y_{2}$ since the same second period income (or social strata) distribution is reached starting from a better distribution of income (or one with more attractive social strata). Formally this is captured by the Upward Structural Mobility (USM) axiom which combines the following properties:

USM.1: For all $i \in N$ and all $y_{1}, y_{2} \in \mathbb{R}_{++}^{n}: M\left(y_{1}, y_{2}^{+\varepsilon(i)}\right)>M\left(y_{1}, y_{2}\right)$.

USM.2: For all $i \in N$ and all $y_{1}, y_{2} \in \mathbb{R}_{++}^{n}: M\left(y_{1}, y_{2}\right)>M\left(y_{1}^{+\varepsilon(i)}, y_{2}\right)$.

USM.1 requires the index to be increasing in its last $n$ arguments, while USM. 2 requires it to be decreasing in its first $n$ arguments. We note that it follows from USM.2 that the measurement of upward structural mobility contradicts the Pareto principle, interpreted to require that an ordering of income vectors is increasing in incomes of both periods. Any social welfare approach to the measurement of mobility ${ }^{3}$ that respects this Pareto principle will never be able to capture the notion of upward structural mobility.

We now consider further structural mobility axioms that change the marginals by changing the degree of inequality in the marginals (or the diversity of the social strata distributions) without affecting the covariance of the incomes in two periods. Given a vector $x=\left(x_{1}, \ldots, x_{i}, \ldots, x_{j}, \ldots x_{n}\right) \in \mathbb{R}_{++}^{n}$, consider $\delta \in \mathbb{R}_{++}$such that $x^{\delta(i j)}=\left(x_{i}, \ldots, x_{i}-\delta, \ldots, x_{j}+\delta, \ldots x_{n}\right) \in \mathbb{R}_{++}^{n}$, which is identical to $x$, except for a transfer $\delta$ that took place from $i$ to $j$. In order to ensure that the covariance between two distributions is not affected by a transfer from $i$ to $j$ in a marginal distribution, we have to impose that the marginal distribution that is not changed belongs to $R_{++}^{n}(i j)$, which, given $i, j \in N$, is defined as $\left\{x \in \mathbb{R}_{++}^{n}: x_{i}=x_{j}\right\}$.

The next axiom is a weak version of Cowell's (1985) Monotonicity in Distance axiom. ${ }^{4}$ The latter states that if second (first) period incomes are further apart than first (second) period incomes, then pushing them even further apart increases mobility. In our context, to make the principle independent of exchange mobility, we impose the restriction that covariances remain unaffected by the considered transfers. Our weak version of the Monotonicity in Distance axiom thus translates into the following Distance Increasing Structural Mobility axiom (DISM):

DISM.1: For all $i, j \in N$ with $y_{2 j} \geq y_{2 i}>\delta \in \mathbb{R}_{++}$, all $y_{1} \in R_{++}^{n}(i j)$ and all $y_{2} \in \mathbb{R}_{++}^{n}: M\left(y_{1}, y_{2}^{\delta(i j)}\right)>M\left(y_{1}, y_{2}\right)$.

${ }^{3}$ This approach has been quite popular (see, e.g., Atkinson, 1981; Markandya, 1982; Chakravarty et al., 1985).

${ }^{4}$ We are grateful to a referee for pointing us to Cowell's (1985) framework. 

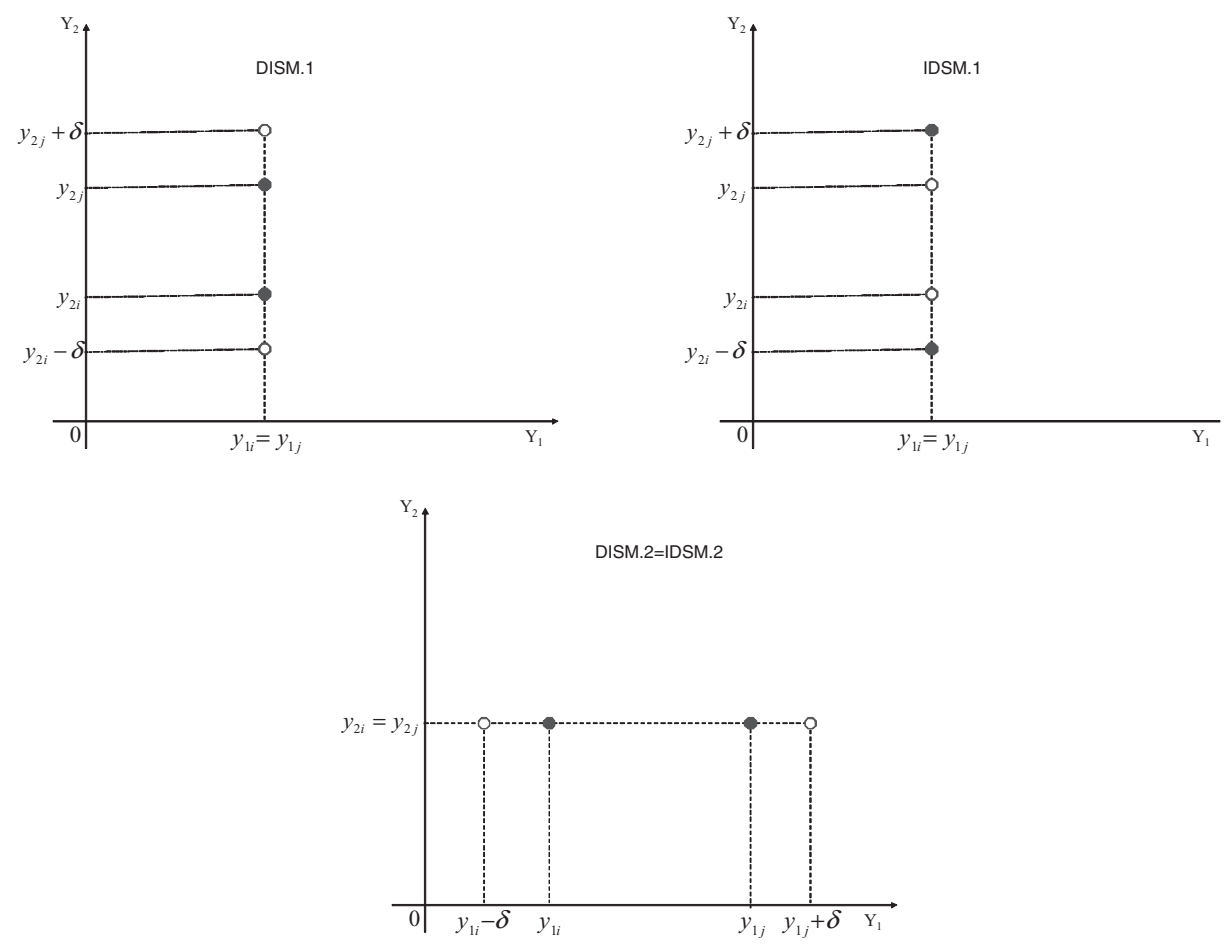

Figure 1. Further Structural Mobility Axioms

DISM.2: For all $i, j \in N$ with $y_{1 j} \geq y_{1 i}>\delta \in \mathbb{R}_{++}$, all $y_{2} \in R_{++}^{n}(i j)$ and all $y_{1} \in \mathbb{R}_{++}^{n}: M\left(y_{1}^{\delta(i j)}, y_{2}\right)>M\left(y_{1}, y_{2}\right)$.

According to the DISM axiom, increasing the inequality in both marginals, while keeping the covariance constant, increases structural mobility. This is illustrated in the top left and bottom panel of Figure 1, where for two observations that have the same income in one period, their incomes in the other period are further apart in the situation with the white dots than in the situation with the black dots. Hence, the move from the black dots to the white dots is distance increasing and so mobility increasing.

Alternatively, one might argue that structural mobility should depend on the extent to which the dynamic process moves toward a more equal distribution. This gives rise to the following Inequality Decreasing Structural Mobility axiom (IDSM):

IDSM.1: For all $i, j \in N$ with $y_{2 j} \geq y_{2 i}>\delta \in \mathbb{R}_{++}$, all $y_{1} \in R_{++}^{n}(i j)$ and all $y_{2} \in \mathbb{R}_{++}^{n}: M\left(y_{1}, y_{2}\right)>M\left(y_{1}, y_{2}^{\delta(i j)}\right)$.

IDSM.2: For all $i, j \in N$ with $y_{1 j} \geq y_{1 i}>\delta \in \mathbb{R}_{++}$, all $y_{2} \in R_{++}^{n}(i j)$ and all $y_{1} \in \mathbb{R}_{++}^{n}: M\left(y_{1}^{\delta(i j)}, y_{2}\right)>M\left(y_{1}, y_{2}\right)$.

The first part follows immediately from the motivation above. The second part captures the fact that in moving from the more unequal $y_{1}^{\delta(i j)}$ rather than $y_{1}$ 
to the same $y_{2}$ we have a larger movement to an equally socially desirable $y_{2}$. The top right and bottom panel of Figure 1 illustrates these properties; the move from the black dots to the white dots makes the process more inequality decreasing and so mobility increasing.

Both the DISM and IDSM axioms seem reasonable, but, as a comparison of the top left and right panels in Figure 1 illustrates, have opposite implications for increasing inequality in the second period income distribution. As a result, they are incompatible and no measure can satisfy both at the same time.

The implications of these axioms for a general mobility index of the form $M\left(y_{1}, y_{2}\right)$ are not clear. In the next section we impose more structure on the mobility index using the RISC axioms.

\section{RESULtS}

Applying insights similar to Foster and Shorrocks (1991) we obtain the following representation result.

Lemma 1: Replication Invariant Subgroup Consistent (RISC) Mobility Measures.

For each $n \geq 1$ and every $\left(y_{1}, y_{2}\right) \in \mathbb{R}_{++}^{2 n}$ a replication invariant subgroup consistent mobility measure can be written as

$$
F\left(\frac{1}{n} \sum_{i=1}^{n} \phi\left(y_{1 i}, y_{2 i}\right)\right)
$$

where $F: \phi\left(\mathbb{R}_{++}^{2}\right) \rightarrow \mathbb{R}$ is continuous and increasing and $\phi: \mathbb{R}_{++}^{2} \rightarrow \mathbb{R}$ is continuous.

Particular subgroup consistent mobility measures have been proposed by Fields and Ok (1996, 1999), namely:

$$
M_{F O_{1}}=\frac{1}{n} \sum_{i=1}^{n}\left|y_{2 i}-y_{1 i}\right|^{\alpha}, M_{F O_{2}}=\frac{1}{n} \sum_{i=1}^{n}\left|\log \left(\frac{y_{2 i}}{y_{1 i}}\right)\right|^{\alpha} \quad \text { and } \quad M_{F O_{3}}=\frac{1}{n} \sum_{i=1}^{n} \log \left(\frac{y_{2 i}}{y_{1 i}}\right)
$$

where $\alpha \in \mathbb{R}_{++}$,

$$
M_{F O_{4}}=\frac{1}{n} \sum_{i=1}^{n}\left|\frac{\left(y_{2 i}\right)^{1-\sigma}}{1-\sigma}-\frac{\left(y_{1 i}\right)^{1-\sigma}}{1-\sigma}\right| \text { for } 0 \leq \sigma \neq 1 \quad \text { and } \quad M_{F O_{4}}=\frac{1}{n} \sum_{i=1}^{n}\left|\log \left(\frac{y_{2 i}}{y_{1 i}}\right)\right| \text { for } \sigma=1 \text {, }
$$

where $\sigma \in \mathbb{R}_{++}$. D'Agostino and Dardanoni (2009) propose

$$
M_{D_{1}}=\frac{1}{n} \sum_{i=1}^{n}\left(y_{1 i}-y_{2 i}\right)^{2} \quad \text { and } \quad M_{D_{2}}=\frac{1}{n} \sum_{i=1}^{n}\left(g\left(y_{1 i}\right)-g\left(y_{2 i}\right)\right)^{2},
$$

where $g($.$) is a continuous and increasing function. { }^{5}$

${ }^{5} \mathrm{Mitra}$ and $\mathrm{Ok}$ (1998) characterize the measure $M_{F O_{1}}$ for values of $\alpha \geq 1$. The range or the value of $\alpha$ is not important for what follows, however. The same is true for the value of $\sigma$ in $M_{F_{4}}$, or the exact shape of the function $g($.$) in M_{D_{2}}$. 
TABLE 1

Some Existing RisC Measures and their Properties

\begin{tabular}{lcccccc}
\hline \hline Measure satisfies axiom? & $M_{F_{1}}$ & $M_{F_{2}}$ & $M_{F O_{3}}$ & $M_{F O_{4}}$ & $M_{D_{1}}$ & $M_{D_{2}}$ \\
\hline EM & $\mathrm{No}^{\mathrm{a}}$ & $\mathrm{No}$ & $\mathrm{No}$ & $\mathrm{No}$ & Yes & Yes \\
USM & $\mathrm{No}$ & $\mathrm{No}$ & $\mathrm{Yes}$ & $\mathrm{No}$ & No & No \\
DISM & $\mathrm{No}^{\mathrm{a}}$ & $\mathrm{No}$ & $\mathrm{No}$ & No & Yes & No \\
IDSM & $\mathrm{No}$ & No & Yes & No & No & No \\
\hline
\end{tabular}

Note: ${ }^{a}$ Except when $\alpha=2$, when the index is equal to $M_{D_{1}}$.

Corollary 1: $M_{\mathrm{FO}_{1}}, M_{\mathrm{FO}_{2}}, M_{\mathrm{FO}_{3}}, M_{\mathrm{FO}_{4}}, M_{D_{1}}$ and $M_{D_{2}}$ are RISC measures of mobility. Their properties in terms of our core axioms (EM and USM) and the other structural mobility axioms (DISM and IDSM) are collected in Table 1.

The measures proposed by D'Agostino and Dardanoni satisfy EM and $M_{D_{1}}$ also satisfies DISM, but only $M_{\mathrm{FO}_{3}}$ satisfies USM. It is worrying that none of these measures satisfies simultaneously EM and USM. ${ }^{6}$

The following corollary shows the restrictions that are imposed by EM, USM, DISM, and IDSM on replication invariant subgroup consistent mobility measures.

Corollary 2: RISC Mobility Measures satisfying EM, USM, DISM, or IDSM.

A replication invariant subgroup consistent mobility measure can be written in the form (1) of Lemma 1. Moreover, it satisfies

(a) EM if and only if for all $y_{1 i}, y_{2 i}, y_{1 j}, y_{2 j} \in \mathbb{R}_{++}^{n}:\left(y_{1 i}-y_{1 j}\right)\left(y_{2 i}-y_{2 j}\right)>0$ $\Rightarrow \phi\left(y_{1 i}, y_{2 i}\right)+\phi\left(y_{1 j}, y_{2 j}\right)<\phi\left(y_{1 i}, y_{2 j}\right)+\phi\left(y_{1 j}, y_{2 i}\right)$.

(b) USM if and only if the function $\phi$ is decreasing in its first argument and increasing in its second argument.

(c) DISM.1 if and only if for all $a, b, c, \delta \in \mathbb{R}_{++}$with $\delta<b<c: \phi(a, b$ $-\delta)-\phi(a, b)>\phi(a, c)-\phi(a, c+\delta)$.

(d) DISM.2 if and only if for all $a, b, c, \delta \in \mathbb{R}_{++}$with $\delta<b<c: \phi(b-\delta, a)$ $-\phi(b, a)>\phi(c, a)-\phi(c+\delta, a)$.

(e) IDSM.1 if and only if for all $a, b, c, \delta \in \mathbb{R}_{++}$with $\delta<b<c: \phi(a$, $b-\delta)-\phi(a, b)<\phi(a, c)-\phi(a, c+\delta)$.

(f) IDSM.2 if and only if for all $a, b, c, \delta \in \mathbb{R}_{++}$with $\delta<b<c: \phi(b-\delta$, $a)-\phi(b, a)>\phi(c, a)-\phi(c+\delta, a)$.

If the cross derivative of the function $\phi$ exists, the condition in part (a) of the Corollary is equivalent to requiring this cross derivative to be negative. ${ }^{7}$ Condition (b) is self evident. If the function $\phi$ is twice differentiable the other conditions reduce to the following: (c) requires that the second derivative with respect to the

${ }^{6}$ Cowell's (1985) measure of distributional change, defined as $\frac{1}{\alpha(\alpha-1)}\left[\sum_{i=1}^{n}\left(\frac{y_{1 i}}{\mu_{1}}\right)^{\alpha}\left(\frac{y_{2 i}}{\mu_{2}}\right)^{1-\alpha}-1\right]$ always satisfies EM and DISM, while it satisfies USM if its sensitivity parameter $\alpha$ is smaller than 0 . This is an interesting measure but it is not subgroup consistent and is therefore not included in Table 1.

${ }^{7}$ In the context of evaluating exchange mobility by an additively separable dynastic social welfare function, this property was already established by Atkinson (1981) and Markandya (1982). 
second argument is positive; (d) and (f) that the second derivative with respect to the first argument is negative; and (e) that the second derivative with respect to the second argument is negative.

A specific functional form for a mobility measure satisfying EM and USM can be obtained by imposing two additional standard axioms for relative mobility measurement:

RSI [Ratio-scale Invariance]: For all $y_{1}, y_{2}, x_{1}, x_{2} \in \mathbb{R}_{++}^{n}$ and for all $\lambda_{1}, \lambda_{2} \in \mathbb{R}_{++}: M\left(y_{1}, y_{2}\right)=M\left(x_{1}, x_{2}\right) \Leftrightarrow M\left(\lambda_{1} y_{1}, \lambda_{2} y_{2}\right)=M\left(\lambda_{1} x_{1}, \lambda_{2} x_{2}\right)$.

SI [Scale Invariance]: For all $y_{1}, y_{2} \in \mathbb{R}_{++}^{n}$ and for all $\lambda \in \mathbb{R}_{++}: M\left(\lambda y_{1}, \lambda y_{2}\right)=$ $M\left(y_{1}, y_{2}\right)$.

We characterize a new relative mobility measure in Theorem 1.

\section{Theorem 1. A new index of relative mobility.}

A replication invariant, subgroup consistent mobility index satisfies EM, USM, $R S I$, and SI if and only if for each $n \geq 1$ and every $\left(y_{1}, y_{2}\right) \in \mathbb{R}_{++}^{2 n}$ it can be written as

$$
F\left(\frac{1}{n} \sum_{i=1}^{n}\left(\frac{y_{2 i}}{y_{1 i}}\right)^{r}\right)
$$

where $F: \phi\left(\mathbb{R}_{++}^{2}\right) \rightarrow \mathbb{R}$ is continuous and increasing and $r \in \mathbb{R}_{++}$. In addition, the measure satisfies DISM if and only if $r>1$ and IDSM if and only if $r<1$.

The class of mobility measures in Theorem 1 is new. A particular subclass, only indexed by $r$, follows by letting $F$ be the identity map,

$$
M(r)=\frac{1}{n} \sum_{i=1}^{n}\left(\frac{y_{2 i}}{y_{1 i}}\right)^{r}
$$

The parameter $r$ has the interpretation of a sensitivity parameter. This becomes plain if $M_{r}$ is rewritten as $\frac{1}{n} \sum_{i=1}^{n}\left(\frac{y_{2 i}}{y_{1 i}}\right)^{r-1}\left(\frac{y_{2 i}}{y_{1 i}}\right)$, where $\left(\frac{y_{2 i}}{y_{1 i}}\right)^{r-1}$ is the weight given to the relative income change of each individual. For $r=1$ all changes get the same weight. If $r<(>) 1$, the weight for small changes is larger (smaller) than for big changes and the measure satisfies IDSM (DISM). Easy to interpret are the values $r=1$, for which all weights are equal and $r=2$, for which the weights are $\left(\frac{y_{2 i}}{y_{1 i}}\right)$. In our empirical illustration we consider several values of $r$ below and above 1 , and examine how rankings change with $r$.

Some of the measures proposed in the literature, such as $M_{F O_{1}}$ and $M_{D_{1}}$, are not relative, but absolute measures of mobility. It is worth pointing out that the present framework can be easily adjusted to characterize an absolute measure of income mobility satisfying the RISC axioms EM and USM by replacing $\mathbb{R}_{++}^{n}$ by $\mathbb{R}^{n}$ in all domain definitions, and after definition of $l$ as an n-dimensional vector of ones, by replacing RSI and SI by, respectively, 
TSI [Translation-scale Invariance]: For all $y_{1}, y_{2}, x_{1}, x_{2} \in \mathbb{R}^{n}$ and for all $\kappa_{1}, \kappa_{2} \in \mathbb{R}: M\left(y_{1}, y_{2}\right)=M\left(x_{1}, x_{2}\right) \Leftrightarrow M\left(y_{1}+\kappa_{1} l, y_{2}+\kappa_{2} l\right)=M\left(x_{1}+\kappa_{1} l, x_{2}+\kappa_{2} l\right)$.

AI [Addition Invariance]: For all $y_{1}, y_{2} \in \mathbb{R}^{n}$ and for all $\kappa \in \mathbb{R}_{++}$: $M\left(y_{1}+\kappa \imath, y_{2}+\kappa \imath\right)=M\left(y_{1}, y_{2}\right)$.

The result is stated in the following theorem.

Theorem 2. A new index of absolute mobility.

A replication invariant, subgroup consistent mobility index satisfies EM, USM, $T S I$, and AI if and only if for each $n \geq 1$ and every $\left(y_{1}, y_{2}\right) \in \mathbb{R}^{2 n}$ it can be written as

$$
F\left(\frac{1}{n} \sum_{i=1}^{n} \exp \left[c\left(y_{2 i}-y_{1 i}\right)\right]\right),
$$

where $F: \phi\left(\mathbb{R}_{++}^{2}\right) \rightarrow \mathbb{R}$ is continuous and increasing and $c \in \mathbb{R}_{++}$. The measure always satisfies DISM.

A particular subclass, only indexed by $c$, follows by letting $F$ be the identity map,

$$
m(c)=\frac{1}{n} \sum_{i=1}^{n} \exp \left[c\left(y_{2 i}-y_{1 i}\right)\right] .
$$

Again $c$ is a sensitivity parameter; larger values of $c$ increase the effect of large absolute income movements. The measure of absolute income mobility always prefers an increase in dispersion in the marginal distributions of both periods, and so satisfies DISM. However to compare the evolution of mobility over time or between countries, relative mobility measures are more attractive, which explains why in the empirical section our focus is on our relative mobility measure (3).

For empirical work, and specifically the empirical illustration that follows, we need to consider the issue of statistical inference for the mobility measure (3). The weak law of large numbers implies that as $n \rightarrow \infty, M(r) \rightarrow E\left(\left(y_{2} / y_{1}\right)^{r}\right)$ and the central limit theorem implies that $M(r)$ is asymptotically distributed as a normal variate with mean $\mu$ and variance $\sigma^{2}: M(r) \sim^{a} N\left(\mu, \sigma^{2}\right)$. Comparing two independent joint distributions, such as in the context of cross-country comparisons, the standardized difference-of-means statistic is asymptotically distributed as a standard normal variate. Similar arguments apply to the measure of absolute mobility $m(c)$.

We therefore have the following:

Lemma 2: Statistical inference for the mobility measures $M(r)$ and $\boldsymbol{m}(\boldsymbol{c})$.

(a) $M(r) \sim^{a} N\left(\mu_{M}, \sigma_{M}^{2}\right)$ with $\mu_{M}=E\left(\left(y_{2} / y_{1}\right)^{r}\right)$ and $n \sigma_{M}^{2}=E\left(\left(y_{2} / y_{1}\right)^{2 r}\right)-$ $\left[E\left(\left(y_{2} / y_{1}\right)^{r}\right)\right]^{2}$. Both $\mu_{M}$ and $\sigma_{M}^{2}$ can be consistently estimated by their sample analogues.

(b) $m(c) \sim^{a} N\left(\mu_{m}, \sigma_{m}^{2}\right)$ with $\mu_{m}=E\left(\exp \left[c\left(y_{2}-y_{1}\right)\right]\right)$ and $n \sigma_{m}^{2}=$ $E\left(\exp \left[2 c\left(y_{2}-y_{1}\right)\right]\right)-\left[E\left(\exp \left[c\left(y_{2}-y_{1}\right)\right]\right)\right]^{2}$. Both $\mu_{m}$ and $\sigma_{m}^{2}$ can be consistently estimated by their sample analogues. 
(c) Consider two independent joint income distributions and the associated mobility measures $M_{1}(r)$ and $M_{2}(r)$ with $M_{1}(r) \sim^{a} N\left(\mu_{M, 1}, \sigma_{M, 1}^{2}\right)$ and $M_{2}(r) \sim^{a} N\left(\mu_{M, 2}, \sigma_{M, 2}^{2}\right)$. Under the null hypothesis that $\mu_{M, 1}=\mu_{M, 2}$ we have $\left[M_{1}(r)-M_{2}(r)\right] /\left[\sigma_{M, 1}^{2}+\sigma_{M, 2}^{2}\right]^{1 / 2} \sim^{a} N(0,1)$.

(d) Consider two independent joint income distributions and the associated mobility measures $m_{1}(c)$ and $m_{2}(c)$. To test the hypothesis that $\mu_{m, 1}=\mu_{m, 2}$ apply $(c)$ with $M$ replaced by $m$.

\section{Empirical Illustration: Income Mobility in the U.S. AND GERMANY REVISITED}

Our empirical application is placed in the context of the ongoing debate about income mobility comparisons between the U.S. and Germany (see, e.g., Burkhauser and Poupore, 1997; Burkhauser et al., 1997; Maasoumi and Trede, 2001; Gottschalk and Spolaore, 2002; Schluter and Trede, 2003; Jenkins and Van Kerm, 2006).

Germany is a useful choice for comparison with the United States: the two countries are the largest and third largest economies but some key institutions differ. In contrast to the United States, the German labor market is characterized by rigidity and relatively centralized wage bargaining. The German social welfare system is much more generous. Hence, the received wisdom is that Germany exhibits both lower income inequality and lower income mobility. Burkhauser and Poupore (1997) and Burkhauser et al. (1997) have observed that when measuring income mobility using Shorrocks (1978) indices Germany is typically ranked more mobile than the U.S., contrary to this received wisdom. Gottschalk and Spolaore (2002) and Schluter and Trede (2003) have advanced some explanation for this surprising ranking. The application of our new measure makes a substantive complementary empirical contribution to this debate since we are able to check whether it mattters to use a distance increasing or inequality decreasing mobility measure. ${ }^{8}$

We follow this empirical literature in the use of data sources, sample selection, and income definitions. The data are from the "Equivalent Data Files" versions of the U.S. Panel Study of Income Dynamics (PSID) and the German SocioEconomic Panel (GSOEP). Both panels are similar in design, and the data provider has generated comparable income variables. In order to be fully comparable to the literature cited above, we consider the same case as Schluter and Trede (2003): the unit of analysis is the person, and the income concept is net (i.e. post-tax post-benefit) income equivalized using the OECD scale (equal to the square root of the household size) in 1996 prices. The period under scrutiny is the years 1984 to 1992, when both countries went through a largely synchronized business cycle, and we consider annual income mobility, i.e. years $t$ and $t+1$. For comparability and statistical robustness, we follow the literature and trim each

\footnotetext{
${ }^{8}$ Moreover, in the light of the discussion in Schluter and Trede (2003) our measure is also transparent about the "local" aspects of mobility, since our measure is subgroup consistent and thus explicit about the aggregation rule for income changes.

${ }^{9} \mathrm{http} / /$ www.human.cornell.edu/che/PAM/Research/Centers-Programs/German-Panel/cnef.cfm.
} 
PSID $(1987,1988)$
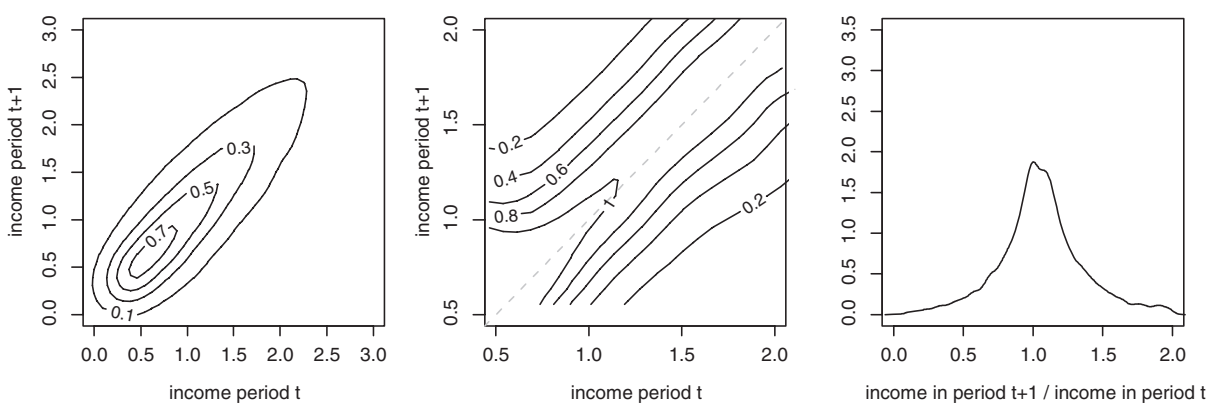

GSOEP(1987,1988)
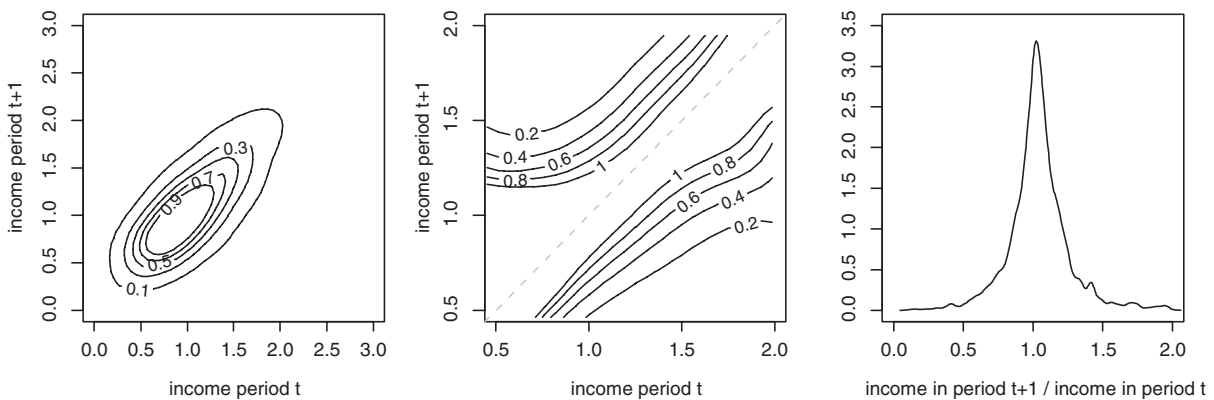

Figure 2. Densities

sample at the 1 percent and 99 percent quantiles. The resulting samples are in excess of 10,000 persons. We are able to replicate the results of cited literature, in particular table 1 of Schluter and Trede (2003): the class of Shorrocks (1978) measures ranks Germany more mobile than the U.S. ${ }^{10}$

Before discussing our point mobility measures we present some descriptive statistics for the period 1987 and 1988; these turn out to be representative for all periods under investigation. In Figure 2, columns 1 and 2, we present contour plots of kernel density estimates of the joint and conditional income distributions (see Schluter (1998) for similar estimates). ${ }^{11}$ The U.S. densities are more dispersed than the German counterparts. A particular (constant) feature of the conditional densities is the greater upward mobility of low-income Germans. Column 3 of the figure depicts the density estimate of relative incomes. This is of interest since the mobility index $M(r)$ transforms relative incomes by means of the function $g(x)=x^{r}$, which is concave if $0<r<1$ and convex for $r \geq 1$. It is evident from the

\footnotetext{
${ }^{11}$ Incomes have been normalized by period $t$ median income for the sake of comparability, the (common) bandwidths have been chosen subjectively, and the conditional density is obtained by simply dividing the joint density estimate by an estimate of the marginal density.
}

${ }^{10}$ Precise details are not reproduced for reasons of brevity but are available on request from the authors. 
TABLE 2

Mobility Comparisons Between the U.S. (PSID) and Germany (GSOEP)

\begin{tabular}{|c|c|c|c|c|c|c|}
\hline Period & $M(0.2)$ & $M(0.4)$ & $M(0.7)$ & $M(1)$ & $M(1.5)$ & $M(2)$ \\
\hline \multicolumn{7}{|c|}{ Panel A: PSID } \\
\hline \multirow[t]{2}{*}{$1984 / 85$} & 1.009 & 1.027 & 1.072 & 1.151 & 1.460 & 2.703 \\
\hline & $(0.0007)$ & $(0.0015)$ & $(0.0035)$ & $(0.0088)$ & $(0.0570)$ & $(0.4268)$ \\
\hline \multirow[t]{2}{*}{$1985 / 86$} & 1.008 & 1.024 & 1.065 & 1.132 & 1.351 & 1.889 \\
\hline & $(0.0007)$ & $(0.0014)$ & $(0.0030)$ & $(0.0058)$ & $(0.0194)$ & $(0.0758)$ \\
\hline \multirow[t]{2}{*}{$1986 / 87$} & 1.008 & 1.023 & 1.062 & 1.126 & 1.330 & 1.811 \\
\hline & $(0.0006)$ & $(0.0014)$ & $(0.0029)$ & $(0.0055)$ & $(0.0173)$ & $(0.0611)$ \\
\hline \multirow[t]{2}{*}{$1987 / 88$} & 1.014 & 1.035 & 1.083 & 1.157 & 1.400 & 2.202 \\
\hline & $(0.0006)$ & $(0.0013)$ & $(0.0030)$ & $(0.0070)$ & $(0.0440)$ & $(0.3444)$ \\
\hline \multirow[t]{2}{*}{$1988 / 89$} & 1.010 & 1.028 & 1.069 & 1.136 & 1.339 & 1.804 \\
\hline & $(0.0006)$ & $(0.0013)$ & $(0.0028)$ & $(0.0054)$ & $(0.0167)$ & $(0.0595)$ \\
\hline \multirow[t]{2}{*}{$1989 / 90$} & 1.002 & 1.012 & 1.040 & 1.090 & 1.250 & 1.628 \\
\hline & $(0.0006)$ & $(0.0013)$ & $(0.0026)$ & $(0.0049)$ & $(0.0175)$ & $(0.0859)$ \\
\hline \multirow[t]{2}{*}{ 1990/91 } & 1.003 & 1.014 & 1.049 & 1.116 & 1.389 & 2.355 \\
\hline & $(0.0007)$ & $(0.0014)$ & $(0.0034)$ & $(0.0078)$ & $(0.0386)$ & $(0.2217)$ \\
\hline \multirow[t]{2}{*}{$1991 / 92$} & 1.002 & 1.013 & 1.045 & 1.104 & 1.311 & 1.861 \\
\hline & $(0.0007)$ & $(0.0014)$ & $(0.0030)$ & $(0.0060)$ & $(0.0213)$ & $(0.0886)$ \\
\hline \multicolumn{7}{|c|}{ Panel B: GSOEP } \\
\hline \multirow[t]{2}{*}{$1984 / 85$} & 1.003 & 1.01 & 1.031 & 1.069 & 1.205 & 1.585 \\
\hline & $(0.0006)$ & $(0.0013)$ & $(0.0029)$ & $(0.0059)$ & $(0.0222)$ & $(0.0945)$ \\
\hline \multirow[t]{2}{*}{$1985 / 86$} & 1.005 & 1.014 & 1.034 & 1.068 & 1.177 & 1.485 \\
\hline & $(0.0006)$ & $(0.0012)$ & $(0.0026)$ & $(0.0054)$ & $(0.0237)$ & $(0.1232)$ \\
\hline \multirow[t]{2}{*}{$1986 / 87$} & 1.015 & 1.033 & 1.066 & 1.109 & 1.211 & 1.374 \\
\hline & $(0.0005)$ & $(0.0011)$ & $(0.0021)$ & $(0.0035)$ & $(0.0081)$ & $(0.0194)$ \\
\hline \multirow[t]{2}{*}{$1987 / 88$} & 1.007 & 1.017 & 1.037 & 1.063 & 1.127 & 1.224 \\
\hline & $(0.0005)$ & $(0.0010)$ & $(0.0018)$ & $(0.0029)$ & $(0.0058)$ & $(0.0118)$ \\
\hline \multirow[t]{2}{*}{$1988 / 89$} & 1.007 & 1.017 & 1.038 & 1.066 & 1.139 & 1.269 \\
\hline & $(0.0005)$ & $(0.0010)$ & $(0.0020)$ & $(0.0035)$ & $(0.0090)$ & $(0.0260)$ \\
\hline \multirow[t]{2}{*}{$1989 / 90$} & 1.004 & 1.011 & 1.025 & 1.047 & 1.101 & 1.192 \\
\hline & $(0.0005)$ & $(0.0010)$ & $(0.0019)$ & $(0.0031)$ & $(0.0065)$ & $(0.0147)$ \\
\hline \multirow[t]{2}{*}{ 1990/91 } & 1.01 & 1.022 & 1.047 & 1.078 & 1.151 & 1.262 \\
\hline & $(0.0005)$ & $(0.0010)$ & $(0.0020)$ & $(0.0031)$ & $(0.0066)$ & $(0.0149)$ \\
\hline \multirow[t]{2}{*}{ 1991/92 } & 1.005 & 1.013 & 1.031 & 1.057 & 1.122 & 1.231 \\
\hline & $(0.0005)$ & $(0.0011)$ & $(0.0021)$ & $(0.0034)$ & $(0.0071)$ & $(0.0159)$ \\
\hline \multicolumn{7}{|c|}{ Panel C: $M_{P S I D}(r)>M_{G S O E P}(r)$} \\
\hline $1984 / 85$ & TRUE & TRUE & TRUE & TRUE & TRUE & TRUE \\
\hline $1985 / 86$ & TRUE & TRUE & TRUE & TRUE & TRUE & TRUE \\
\hline $1986 / 87$ & FALSE & FALSE & FALSE $^{n s}$ & TRUE & TRUE & TRUE \\
\hline $1987 / 88$ & TRUE & TRUE & TRUE & TRUE & TRUE & TRUE \\
\hline $1988 / 89$ & TRUE & TRUE & TRUE & TRUE & TRUE & TRUE \\
\hline $1989 / 90$ & FALSE & TRUE ${ }^{n s}$ & TRUE & TRUE & TRUE & TRUE \\
\hline $1990 / 91$ & FALSE & FALSE & TRUE $^{n s}$ & TRUE & TRUE & TRUE \\
\hline $1991 / 92$ & FALSE & FALSE $^{n s}$ & TRUE & TRUE & TRUE & TRUE \\
\hline
\end{tabular}

Notes: SE in parenthesis. In Panel 3 "ns" denotes not statistically significant at 5\% level.

plots that the U.S. density has far more mass in both tails than the density for Germany. Hence we expect that the $M(r)$ index ranks the U.S. more mobile than Germany for $r \geq 1$, but this ranking could be reversed for $r$ sufficiently small to compensate for the higher U.S. mean.

We proceed to apply our mobility measure (3) for increasing values of the sensitivity parameter $r \in\{0.2,0.4,0.7,1,1.5,2\}$. The results are reported in Table 2. In particular, Panels $\mathrm{A}$ and $\mathrm{B}$ report the point measures as well as the estimated standard errors. Panel $\mathrm{C}$ summarizes the results, reporting whether the 
U.S. is ranked more mobile than Germany, and indicates if the ranking is statistically not significant.

As expected from the plot of the relative income densities the measure $M(r)$ ranks the U.S. more mobile than Germany for all values of $r \geq 1$ and all these differences are statistically significant. For smaller values of $r$ the U.S. is ranked more mobile than Germany for many but not all years; prominent exceptions are the periods 1986/87 and 1990/91 and, as expected, these exceptions become more prominent for smaller values of $r$. We conclude that the U.S. typically exhibits more upward structural mobility than Germany. Mobility rankings based on the Shorrocks (1978) class do not capture structural mobility.

Next, we illustrate the usefulness of decompositions by population subgroups. The mobility index for group $g$ is given by

$$
M^{g}(r)=\frac{1}{n_{g}} \sum_{i \in I_{g}}\left(\frac{y_{2, i}}{y_{1, i}}\right)^{r},
$$

where $I_{g}$ denotes the set of individuals in group $g$ which is of size $n_{g}$. The overall index is thus $M(r)=\sum_{g}\left(n_{g} / n\right) M^{g}(r)$. The first decomposition exercise fixes a country, and then compares $M^{g}(r)$ across the various groups. In the second exercises we compare $M^{g}(r)$ for each $g$ and $r$ across countries.

We turn to our empirical implementation. We consider the period 1991/92, a year in which the overall mobility ranking between the U.S. and Germany depends on the value of $r$, and focus on a partition induced by household type in 1991: group 1 consists of single person households with no children, group 2 of single parents with children, group 3 of multi-person households without children, and group 4 of parents with children. Table 3 reports the respective population shares and the mobility estimates. For brevity's sake we report only point estimates.

TABLE 3

Mobility Comparisons Between the U.S. (PSID) and Germany (GSOEP) by Household Type FOR THE PERIOD 1991/92

\begin{tabular}{|c|c|c|c|c|c|c|c|}
\hline Group & Pop. Share & $M(0.2)$ & $M(0.4)$ & $M(0.7)$ & $M(1)$ & $M(1.5)$ & $M(2)$ \\
\hline \multicolumn{8}{|c|}{ Panel A: PSID } \\
\hline 1 & 0.09 & 1.004 & 1.022 & 1.078 & 1.184 & 1.585 & 2.729 \\
\hline 2 & 0.10 & 1.005 & 1.019 & 1.061 & 1.133 & 1.350 & 1.789 \\
\hline 3 & 0.24 & 1.000 & 1.007 & 1.037 & 1.091 & 1.270 & 1.683 \\
\hline 4 & 0.57 & 1.003 & 1.012 & 1.041 & 1.092 & 1.278 & 1.813 \\
\hline \multicolumn{8}{|c|}{ Panel B: GSOEP } \\
\hline 1 & 0.09 & 1.012 & 1.028 & 1.062 & 1.111 & 1.240 & 1.475 \\
\hline 2 & 0.02 & 1.022 & 1.050 & 1.102 & 1.169 & 1.320 & 1.539 \\
\hline 3 & 0.37 & 1.005 & 1.012 & 1.030 & 1.057 & 1.128 & 1.251 \\
\hline 4 & 0.52 & 1.004 & 1.011 & 1.026 & 1.048 & 1.099 & 1.178 \\
\hline \multicolumn{8}{|c|}{ Panel C: $\quad M_{P S I D}^{g}(r)>M_{G S O E P}^{g}(r)$} \\
\hline 1 & & FALSE & FALSE & TRUE & TRUE & TRUE & TRUE \\
\hline 2 & & FALSE & FALSE & FALSE & FALSE & TRUE & TRUE \\
\hline 3 & & FALSE & FALSE & TRUE & TRUE & TRUE & TRUE \\
\hline 4 & & FALSE & TRUE & TRUE & TRUE & TRUE & TRUE \\
\hline
\end{tabular}

Notes: Group 1: single parent with no children; Group 2: single parent with children; Group 3: Not single without children; Group 4: Not single with children. 
In both countries groups 1 and 2 are more mobile than groups 3 and 4 . The remaining rankings, however, are country specific. In Germany group 2 is more mobile than group 1, while in the U.S. group 1 is more mobile than group 2 except for the lowest value of $r$. Group 3 is more mobile than group 4 in Germany while in the U.S. the opposite applies. The ranking of different household types therefore does not depend substantially on the value for $r$ for either country. One explanation for the comparatively high mobility of single households is the fact that a substantial proportion leave this household type category in the subsequent year. In Germany, a quarter of single parents change type (the corresponding share for the other groups is 5 percent), in the U.S. 12 percent of group 1 and 15 percent of group 2 change type. The comparison between the U.S. and Germany for each household type broadly reflects the economy-wide mobility ranking reported in Table 2. For high values of $r$ the U.S. dominates Germany for all household types, but the ranking is reversed as $r$ decreases.

\section{CONCLUSIONS}

We have derived axiomatically new mobility measures of upward structural mobility which respect the notion of exchange mobility, and developed methods of statistical inference. We have illustrated the usefulness of the measure by considering the ongoing debate about mobility comparisons between the U.S. and Germany. Our substantive empirical contribution is the insight that the U.S. exhibits typically greater upward structural mobility, and hence the proposed measure $M(r)$ ranks the U.S. typically more mobile than Germany. The empirical literature has employed measures which do not respect these notions.

We conclude by the methodological observation that some researchers (see, e.g., Ruiz-Castillo, 2004; Van Kerm, 2004) seek to decompose overall mobility into a structural and exchange mobility component. When one wants more upward (downward) structural mobility to influence the index positively (negatively) such a decomposition should be based on a mobility index that satisfies both the EM and USM axioms. Our measures are therefore prime candidates for such decomposition exercises.

\section{APPENDIX: ProOFS}

Proof of Lemma 1. The proof of this lemma follows Foster and Shorrocks' (1991) proof of their proposition 1. For completeness it is repeated here. We first establish that the mobility measure needs to be separable, defined as follows.

SEP [Separability]: For all $y_{1}^{1}, y_{1}^{2}, y_{2}^{1}, y_{2}^{2}, y_{1}^{1 \prime}, y_{1}^{2 \prime}, y_{2}^{1 \prime}, y_{2}^{2 \prime} \in \mathbb{R}_{++}^{n}$ and for all $P \in \mathcal{P}$ : $M\left(y_{1}^{1}, y_{1}^{2}, y_{2}^{1}, y_{2}^{2}\right) \geq M\left(y_{1}^{1 \prime}, y_{1}^{2}, y_{2}^{1 \prime}, y_{2}^{2}\right) \Rightarrow M\left(y_{1}^{1}, y_{1}^{2 \prime}, y_{2}^{1}, y_{2}^{2 \prime}\right) \geq M\left(y_{1}^{1 \prime}, y_{1}^{2 \prime}, y_{2}^{1 \prime}, y_{2}^{2 \prime}\right)$.

Result 1. If a mobility measure is subgroup consistent and symmetric then it also satisfies SEP. 
Proof. Suppose $M\left(y_{1}^{1}, y_{1}^{2}, y_{2}^{1}, y_{2}^{2}\right) \geq M\left(y_{1}^{1 \prime}, y_{1}^{2}, y_{2}^{1 \prime}, y_{2}^{2}\right)$. Then, by subgroup consistency we must have that $M\left(y_{1}^{1}, y_{2}^{1}\right) \geq M\left(y_{1}^{1 \prime}, y_{2}^{1 \prime}\right)$.

(a) If $M\left(y_{1}^{1}, y_{2}^{1}\right)>M\left(y_{1}^{1 \prime}, y_{2}^{1 \prime}\right)$, then immediately by subgroup consistency

$$
M\left(y_{1}^{1}, y_{1}^{2 \prime}, y_{2}^{1}, y_{2}^{2 \prime}\right)>M\left(y_{1}^{1 \prime}, y_{1}^{2 \prime}, y_{2}^{1 \prime}, y_{2}^{2 \prime}\right) .
$$

(b) If $M\left(y_{1}^{1}, y_{2}^{1}\right)=M\left(y_{1}^{1 \prime}, y_{2}^{1 \prime}\right)$, we cannot have that $M\left(y_{1}^{1}, y_{1}^{2 \prime}, y_{2}^{1}, y_{2}^{2 \prime}\right)<$ $M\left(y_{1}^{1 \prime}, y_{1}^{2 \prime}, y_{2}^{1 \prime}, y_{2}^{2 \prime}\right)$, because this would imply by subgroup consistency that $M\left(y_{1}^{1}, y_{1}^{2 \prime}, y_{1}^{1 \prime}, y_{2}^{1}, y_{2}^{2 \prime}, y_{2}^{1 \prime}\right)<M\left(y_{1}^{1 \prime}, y_{1}^{2 \prime}, y_{1}^{1}, y_{2}^{1 \prime}, y_{2}^{2 \prime}, y_{2}^{1}\right)$, which contradicts the anonymity of $M\left(y_{1}, y_{2}\right)$.

Returning to the proof of Lemma 1 , let $z_{i}=\left[y_{1 i}, y_{2 i}\right]$. Then, using Result 1 , due to the fact that, by definition, $M\left(y_{1}, y_{2}\right)$ is not constant on $D$ and a standard result on separability (due to Gorman, 1968), structural mobility, subgroup consistent, anonymous and continuous mobility measures can be written in the following form for any integer $n \geq 3$ :

$$
M\left(y_{1}, y_{2}\right)=\tilde{F}_{n}\left(\sum_{i=1}^{n} \tilde{\phi}_{n}\left(y_{1 i}, y_{2 i}\right)\right) \text { for all }\left(y_{1}, y_{2}\right) \in \mathbb{R}_{++}^{2 n},
$$

where $\tilde{F}_{n}$ is continuous and increasing and $\tilde{\phi}_{n}$ is continuous.

Next, define $\quad \phi_{n}(t) \equiv n\left[\tilde{\phi}_{n}(t)-\tilde{\phi}_{n}(v)\right] \quad$ for $\quad t \in \mathbb{R}_{++}^{2} \Rightarrow \tilde{\phi}_{n}(t)=$ $\frac{1}{n} \phi_{n}(t)+\tilde{\phi}_{n}(v), \Rightarrow \sum_{i=1}^{n} \tilde{\phi}_{n}\left(z_{i}\right)=\frac{1}{n} \sum_{i=1}^{n} \phi_{n}\left(z_{i}\right)+n \tilde{\phi}_{n}(v)$. Define $F_{n}(u) \equiv \tilde{F}_{n}\left(u+n \tilde{\phi}_{n}(v)\right)$ for all $u \in \phi_{n}\left(\mathbb{R}_{++}^{2}\right)$, hence

$$
\tilde{F}_{n}\left(\sum_{i=1}^{n} \tilde{\phi}_{n}\left(z_{i}\right)\right)=\tilde{F}_{n}\left(\frac{1}{n} \sum_{i=1}^{n} \phi_{n}\left(z_{i}\right)+n \tilde{\phi}_{n}(v)\right)=F_{n}\left(\frac{1}{n} \sum_{i=1}^{n} \phi_{n}\left(z_{i}\right)\right),
$$

such that we can write subgroup consistent, anonymous and continuous mobility measures as

$$
M\left(y_{1}, y_{2}\right)=F_{n}\left(\frac{1}{n} \sum_{i=1}^{n} \phi_{n}\left(y_{1 i}, y_{2 i}\right)\right) \text { for all } n \geq 3 \text { and }\left(y_{1}, y_{2}\right) \in \mathbb{R}_{++}^{2 n},
$$

where $F_{n}: \phi_{n}\left(\mathbb{R}_{++}^{2}\right) \rightarrow \mathbb{R}$ is continuous and increasing and $\phi_{n}: \mathbb{R}_{++}^{2} \rightarrow \mathbb{R}$ is continuous.

Note that replication invariance allows us to choose the functions $\phi_{n}$ and $F_{n}$ to be independent of $n$, and extend the formula to the cases where $n=1$ and $n=2$. This is shown next.

Take a particular 2-dimensional vector $t$ and replicate it 4 times to obtain the vector $w$. Denote the $m=4 n$ (with $n$ a positive integer) times replication of vector $t$ by $w^{\prime}$. Define $\phi \equiv \phi_{4}$ and $F \equiv F_{4}$. By RISC. $2, F_{m}\left(\phi_{m}(t)\right)=M\left(w^{\prime}\right)=M(w)=F(\phi(t))$ for every $t \in \mathbb{R}_{++}^{2}$, such that $\phi_{m}(t)=F_{m}^{-1}(F(\phi(t)))$.

Consequently, mobility for $\left(y_{1}, y_{2}\right)$ with $y_{1}$ and $y_{2}$ of dimension $m$ becomes $M\left(y_{1}, y_{2}\right)=F_{m}\left(\frac{1}{m} \sum_{i=1}^{m} \phi_{m}\left(z_{i}\right)\right)=F_{m}\left(\frac{1}{m} \sum_{i=1}^{m} F_{m}^{-1}\left(F\left(\phi\left(z_{i}\right)\right)\right)\right)$, and so $F^{-1}\left(M\left(y_{1}, y_{2}\right)\right)=$ $F^{-1}\left(F_{m}\left(\frac{1}{m} \sum_{i=1}^{m} F_{m}^{-1}\left(F\left(\phi\left(z_{i}\right)\right)\right)\right)\right)$, which can be written as 


$$
F^{-1}\left(M\left(y_{1}, y_{2}\right)\right)=G_{m}^{-1}\left(\frac{1}{m} \sum_{i=1}^{m} G_{m}\left(\phi\left(y_{1 i}, y_{2 i}\right)\right)\right)
$$

after defining $G_{m}^{-1}(u)=F^{-1}\left(F_{m}(u)\right)$, a continuous and increasing function on $\phi\left(\mathbb{R}_{++}^{2}\right)$ and since $F=F_{4}, G_{4}(u)=u$.

Now consider any $\left(y_{1}, y_{2}\right) \in \mathbb{R}_{++}^{2}$ and its replications $\left(y_{1}^{\prime}, y_{2}^{\prime}\right) \in \mathbb{R}_{++}^{4 \cdot 2}$ and $\left(y_{1}^{\prime \prime}, y_{2}^{\prime \prime}\right) \in \mathbb{R}_{++}^{m \cdot 2}$. Define $u_{1}=\phi\left(z_{1}\right)=\phi\left(y_{11}, y_{21}\right)$ and $u_{2}=\phi\left(z_{2}\right)=\phi\left(y_{12}, y_{22}\right)$. We then have from (A1)

$$
F^{-1}\left(M\left(y_{1}^{\prime \prime}, y_{2}^{\prime \prime}\right)\right)=G_{m}^{-1}\left(\frac{1}{m} \sum_{i=1}^{m} G_{m}\left(\phi\left(z_{i}^{\prime \prime}\right)\right)\right)=G_{m}^{-1}\left(\frac{1}{2} G_{m}\left(u_{1}\right)+\frac{1}{2} G_{m}\left(u_{2}\right)\right) \text { and from, }
$$

first RISC.2 and then (A1) again,

$$
F^{-1}\left(M\left(y_{1}^{\prime \prime}, y_{2}^{\prime \prime}\right)\right)=F_{m}^{-1}\left(M\left(y_{1}^{\prime}, y_{2}^{\prime}\right)\right)=G_{4}^{-1}\left(\frac{1}{2} G_{4}\left(u_{1}\right)+\frac{1}{2} G_{4}\left(u_{2}\right)\right)=\frac{1}{2}\left(u_{1}+u_{2}\right),
$$

such that since both LHS are equal,

$$
\left(\frac{1}{2} G_{m}\left(u_{1}\right)+\frac{1}{2} G_{m}\left(u_{2}\right)\right)=G_{m}\left(\frac{1}{2}\left(u_{1}+u_{2}\right)\right) \text { for all } u_{1}, u_{2} \in \phi\left(\mathbb{R}_{++}^{2}\right) \text {. }
$$

The solution to this Jensen equation (Aczél, 1966, p. 46) implies $G_{m}(u)=r_{m} u+s_{m}$ for some constants $r_{m}$ and $s_{m}$, such that

$$
\begin{aligned}
& F^{-1}\left(M\left(y_{1}, y_{2}\right)\right)=G_{m}^{-1}\left(\frac{1}{m} \sum_{i=1}^{m} G_{m}\left(\phi\left(z_{i}\right)\right)\right) \\
& =\frac{1}{r_{m}}\left[\frac{1}{m} \sum_{i=1}^{m}\left(r_{m} \phi\left(z_{i}\right)+s_{m}\right)-s_{m}\right] \\
& =\frac{1}{r_{m}}\left[r_{m} \frac{1}{m} \sum_{i=1}^{m} \phi\left(z_{i}\right)+s_{m}-s_{m}\right] \\
& =\frac{1}{m} \sum_{i=1}^{m} \phi\left(z_{i}\right) .
\end{aligned}
$$

Hence we have

$$
F^{-1}\left(M\left(y_{1}, y_{2}\right)\right)=\frac{1}{m} \sum_{i=1}^{m} \phi\left(y_{1 i}, y_{2 i}\right) \text { for all }\left(y_{1}, y_{2}\right) \in \mathbb{R}_{++}^{m \cdot 2}
$$

whenever $m=4 n$ and $n$ is a positive integer.

Finally, for each $n \geq 1$ consider any $\left(y_{1}, y_{2}\right) \in \mathbb{R}_{++}^{2 n}$ and its replication $\left(y_{1}^{\prime}, y_{2}^{\prime}\right) \in \mathbb{R}_{++}^{4 \cdot 2 n}$. By RISC. 2 and the last equation, we obtain $F^{-1}\left(M\left(y_{1}, y_{2}\right)\right)=$ $F^{-1}\left(M\left(y_{1}^{\prime}, y_{2}^{\prime}\right)\right)=\frac{1}{4 n} \sum_{i=1}^{4 n} \phi\left(z_{i}^{\prime}\right)=\frac{1}{n} \sum_{i=1}^{n} \phi\left(z_{i}\right)$, such that

$$
M\left(y_{1}, y_{2}\right)=F\left(\frac{1}{n} \sum_{i=1}^{n} \phi\left(y_{1 i}, y_{2 i}\right)\right) \text { for each } n \geq 1 \text { and every }\left(y_{1}, y_{2}\right) \in \mathbb{R}_{++}^{2 n},
$$


where $F: \phi\left(\mathbb{R}_{++}^{2}\right) \rightarrow \mathbb{R}$ is continuous and increasing and $\phi: \mathbb{R}_{++}^{2} \rightarrow \mathbb{R}$ is continuous.

Proof of Corollary 2. The proof follows directly from Lemma 1 and axioms EM, USM, DISM and IDSM.

Proof of Theorem 1. The following proof is a completed version ${ }^{12}$ of the proof of theorem 1 in Tsui (1995). We first need two lemmas.

Lemma 2. $M\left(y_{1}, y_{2}\right) \geq M\left(y_{1}^{\prime}, y_{2}^{\prime}\right)$ if and only if, for all $\lambda_{1}, \lambda_{2} \in \mathbb{R}_{++}$: $M\left(\lambda_{1} y_{1}, \lambda_{2} y_{2}\right) \geq M\left(\lambda_{1} y_{1}^{\prime}, \lambda_{2} y_{2}^{\prime}\right)$.

Proof. Assume on the contrary that

$$
M\left(y_{1}, y_{2}\right) \geq(\leq) M\left(y_{1}^{\prime}, y_{2}^{\prime}\right) \text { and } M\left(\lambda_{1} y_{1}, \lambda_{2} y_{2}\right)<(>) M\left(\lambda_{1} y_{1}^{\prime}, \lambda_{2} y_{2}^{\prime}\right) .
$$

The function $M\left(\delta_{1} y_{1}, \delta_{2} y_{2}\right)$ is a continuous function of $\delta_{1}$ and $\delta_{2} \in \mathbb{R}_{++}$. By assumption the image of the function contains positive and negative values. Since the function is continuous, by the intermediate value theorem, there must exist numbers $\gamma_{1}$ and $\gamma_{2} \in \mathbb{R}_{++}$such that $M\left(\gamma_{1} y_{1}, \gamma_{2} y_{2}\right)=M\left(\gamma_{1} y_{1}^{\prime}, \gamma_{2} y_{2}^{\prime}\right)$.

Using scale invariance twice,

$$
M\left(\lambda_{1} y_{1}, \lambda_{2} y_{2}\right)=M\left(\gamma_{1} y_{1}, \gamma_{2} y_{2}\right) \quad \text { and } \quad M\left(\lambda_{1} y_{1}^{\prime}, \lambda_{2} y_{2}^{\prime}\right)=M\left(\gamma_{1} y_{1}^{\prime}, \gamma_{2} y_{2}^{\prime}\right)
$$

such that $M\left(\lambda_{1} y_{1}, \lambda_{2} y_{2}\right)=M\left(\lambda_{1} y_{1}^{\prime}, \lambda_{2} y_{2}^{\prime}\right)$, a contradiction.

Lemma 3. For all $a_{1}, a_{2}, b_{1}, b_{2}, d_{1}, d_{2}, e_{1}, e_{2} \in \mathbb{R}_{++}$and all $\lambda_{1}, \lambda_{2} \in \mathbb{R}_{++}$:

$$
\frac{\phi\left(\lambda_{1} a_{1}, \lambda_{2} a_{2}\right)-\phi\left(\lambda_{1} b_{1}, \lambda_{2} b_{2}\right)}{\phi\left(a_{1}, a_{2}\right)-\phi\left(b_{1}, b_{2}\right)}=\frac{\phi\left(\lambda_{1} d_{1}, \lambda_{2} d_{2}\right)-\phi\left(\lambda_{1} e_{1}, \lambda_{2} e_{2}\right)}{\phi\left(d_{1}, d_{2}\right)-\phi\left(e_{1}, e_{2}\right)}
$$

Proof. Consider eight elements of $\mathbb{R}_{++}: a_{1}, a_{2}, b_{1}, b_{2}, d_{1}, d_{2}, e_{1}$ and $e_{2}$ and define the real number $k$ such that:

$$
\phi\left(d_{1}, d_{2}\right)-\phi\left(e_{1}, e_{2}\right)=k\left(\phi\left(a_{1}, a_{2}\right)-\phi\left(b_{1}, b_{2}\right)\right) .
$$

Case 1: $k$ is a rational number. Consider two natural numbers $K$ and $L$, such that $|k|=K / L$.

(a) If $k=K / L$, define $n=K+L$ and rewrite equation (A3) as:

$$
\frac{K}{n} \phi\left(a_{1}, a_{2}\right)+\frac{L}{n} \phi\left(e_{1}, e_{2}\right)=\frac{K}{n} \phi\left(b_{1}, b_{2}\right)+\frac{L}{n} \phi\left(d_{1}, d_{2}\right) .
$$

Using ratio-scale invariance, this implies

\footnotetext{
${ }^{12} \mathrm{We}$ are grateful to Thomas Demuynck for the proof of Lemma 3.
} 


$$
\frac{K}{n} \phi\left(\lambda_{1} a_{1}, \lambda_{2} a_{2}\right)+\frac{L}{n} \phi\left(\lambda_{1} e_{1}, \lambda_{2} e_{2}\right)=\frac{K}{n} \phi\left(\lambda_{1} b_{1}, \lambda_{2} b_{2}\right)+\frac{L}{n} \phi\left(\lambda_{1} d_{1}, \lambda_{2} d_{2}\right) .
$$

Using the last two equations,

$$
\frac{\phi\left(\lambda_{1} a_{1}, \lambda_{2} a_{2}\right)-\phi\left(\lambda_{1} b_{1}, \lambda_{2} b_{2}\right)}{\phi\left(\lambda_{1} d_{1}, \lambda_{2} d_{2}\right)-\phi\left(\lambda_{1} e_{1}, \lambda_{2} e_{2}\right)}=\frac{1}{k}=\frac{\phi\left(a_{1}, a_{2}\right)-\phi\left(b_{1}, b_{2}\right)}{\phi\left(d_{1}, d_{2}\right)-\phi\left(e_{1}, e_{2}\right)},
$$

which after exchanging the numerator and denominator yields (A2).

(b) If $k=-K / L$, define $n=K+L$ and derive from (A3) that

$$
\frac{K}{n} \phi\left(a_{1}, a_{2}\right)+\frac{L}{n} \phi\left(d_{1}, d_{2}\right)=\frac{K}{n} \phi\left(b_{1}, b_{2}\right)+\frac{L}{n} \phi\left(e_{1}, e_{2}\right) .
$$

Proceeding in the same way as under (a) allows us to show (A2).

Case 2: $k$ is an irrational number.

(a) Suppose that $\phi\left(a_{1}, a_{2}\right)-\phi\left(b_{1}, b_{2}\right)>0$.

(i) Suppose that $k>0$. Consider two strictly positive arbitrary rational numbers $r_{1}$ and $r_{2}$ such that $r_{1}<k<r_{2}$. Consequently, using (A3),

$$
r_{1}\left(\phi\left(a_{1}, a_{2}\right)-\phi\left(b_{1}, b_{2}\right)\right)<\phi\left(d_{1}, d_{2}\right)-\phi\left(e_{1}, e_{2}\right)<r_{2}\left(\phi\left(a_{1}, a_{2}\right)-\phi\left(b_{1}, b_{2}\right)\right) .
$$

Let $r_{1}=K_{1} / L_{1}$ and $r_{2}=K_{2} / L_{2}$. Defining $n_{1}=K_{1}+L_{1}$ and $n_{2}=K_{2}+L_{2}$, we derive that

$$
\begin{aligned}
& \frac{K_{1}}{n_{1}} \phi\left(a_{1}, a_{2}\right)+\frac{L_{1}}{n_{1}} \phi\left(e_{1}, e_{2}\right)<\frac{K_{1}}{n_{1}} \phi\left(b_{1}, b_{2}\right)+\frac{L_{1}}{n_{1}} \phi\left(d_{1}, d_{2}\right), \\
& \frac{K_{2}}{n_{2}} \phi\left(a_{1}, a_{2}\right)+\frac{L_{2}}{n_{2}} \phi\left(e_{1}, e_{2}\right)>\frac{K_{2}}{n_{2}} \phi\left(b_{1}, b_{2}\right)+\frac{L_{2}}{n_{2}} \phi\left(d_{1}, d_{2}\right) .
\end{aligned}
$$

Due to Lemma 2, these inequalities imply

$$
\begin{aligned}
& \frac{K_{1}}{n_{1}} \phi\left(\lambda_{1} a_{1}, \lambda_{2} a_{2}\right)+\frac{L_{1}}{n_{1}} \phi\left(\lambda_{1} e_{1}, \lambda_{2} e_{2}\right)<\frac{K_{1}}{n_{1}} \phi\left(\lambda_{1} b_{1}, \lambda_{2} b_{2}\right)+\frac{L_{1}}{n_{1}} \phi\left(\lambda_{1} d_{1}, \lambda_{2} d_{2}\right), \\
& \frac{K_{2}}{n_{2}} \phi\left(\lambda_{1} a_{1}, \lambda_{2} a_{2}\right)+\frac{L_{2}}{n_{2}} \phi\left(\lambda_{1} e_{1}, \lambda_{2} e_{2}\right)>\frac{K_{2}}{n_{2}} \phi\left(\lambda_{1} b_{1}, \lambda_{2} b_{2}\right)+\frac{L_{2}}{n_{2}} \phi\left(\lambda_{1} d_{1}, \lambda_{2} d_{2}\right) .
\end{aligned}
$$

Rearranging these equations we have that

$$
r_{1}=\frac{K_{1}}{L_{1}}<\frac{\phi\left(\lambda_{1} d_{1}, \lambda_{2} d_{2}\right)-\phi\left(\lambda_{1} e_{1}, \lambda_{2} e_{2}\right)}{\phi\left(\lambda_{1} a_{1}, \lambda_{2} a_{2}\right)-\phi\left(\lambda_{1} b_{1}, \lambda_{2} b_{2}\right)}<\frac{K_{2}}{L_{2}}=r_{2} .
$$

We proceed now to show that 


$$
\frac{\phi\left(\lambda_{1} d_{1}, \lambda_{2} d_{2}\right)-\phi\left(\lambda_{1} e_{1}, \lambda_{2} e_{2}\right)}{\phi\left(\lambda_{1} a_{1}, \lambda_{2} a_{2}\right)-\phi\left(\lambda_{1} b_{1}, \lambda_{2} b_{2}\right)}=k
$$

Suppose this equality does not hold. In that case, there exists a rational number $r_{1}^{\prime}>0$ or a rational number $r_{2}^{\prime}>0$ such that either

$$
\frac{\phi\left(\lambda_{1} d_{1}, \lambda_{2} d_{2}\right)-\phi\left(\lambda_{1} e_{1}, \lambda_{2} e_{2}\right)}{\phi\left(\lambda_{1} a_{1}, \lambda_{2} a_{2}\right)-\phi\left(\lambda_{1} b_{1}, \lambda_{2} b_{2}\right)}<r_{1}^{\prime}<k, \text { or } \frac{\phi\left(\lambda_{1} d_{1}, \lambda_{2} d_{2}\right)-\phi\left(\lambda_{1} e_{1}, \lambda_{2} e_{2}\right)}{\phi\left(\lambda_{1} a_{1}, \lambda_{2} a_{2}\right)-\phi\left(\lambda_{1} b_{1}, \lambda_{2} b_{2}\right)}>r_{2}^{\prime}>k
$$

However, since the rational numbers leading to (A4) were arbitrarily chosen, (A4) applies for $r_{1}^{\prime}$ and $r_{2}^{\prime}$, such that we must have

$$
r_{1}^{\prime}<\frac{\phi\left(\lambda_{1} d_{1}, \lambda_{2} d_{2}\right)-\phi\left(\lambda_{1} e_{1}, \lambda_{2} e_{2}\right)}{\phi\left(\lambda_{1} a_{1}, \lambda_{2} a_{2}\right)-\phi\left(\lambda_{1} b_{1}, \lambda_{2} b_{2}\right)}<r_{2}^{\prime}
$$

which contradicts (A6), such that (A5) must hold true. Combining (A5) with (A3) proofs (A2).

(ii) Suppose that $k<0$. Consider two strictly negative arbitrary rational numbers $r_{1}$ and $r_{2}$ such that $r_{1}<k<r_{2}$. Consequently, using (A3),

$$
r_{1}\left(\phi\left(a_{1}, a_{2}\right)-\phi\left(b_{1}, b_{2}\right)\right)<\phi\left(d_{1}, d_{2}\right)-\phi\left(e_{1}, e_{2}\right)<r_{2}\left(\phi\left(a_{1}, a_{2}\right)-\phi\left(b_{1}, b_{2}\right)\right) .
$$

Let $r_{1}=-K_{1} / L_{1}$ and $r_{2}=-K_{2} / L_{2}$. Defining $n_{1}=K_{1}+L_{1}$ and $n_{2}=K_{2}+L_{2}$, we derive that

$$
\begin{aligned}
& \frac{K_{1}}{n_{1}} \phi\left(a_{1}, a_{2}\right)+\frac{L_{1}}{n_{1}} \phi\left(d_{1}, d_{2}\right)>\frac{K_{1}}{n_{1}} \phi\left(b_{1}, b_{2}\right)+\frac{L_{1}}{n_{1}} \phi\left(e_{1}, e_{2}\right), \\
& \frac{K_{2}}{n_{2}} \phi\left(a_{1}, a_{2}\right)+\frac{L_{2}}{n_{2}} \phi\left(d_{1}, d_{2}\right)<\frac{K_{2}}{n_{2}} \phi\left(b_{1}, b_{2}\right)+\frac{L_{2}}{n_{2}} \phi\left(e_{1}, e_{2}\right) .
\end{aligned}
$$

Repeating the same steps as in (i), it is easy to show that (A2) holds true.

(b) Suppose that $\phi\left(a_{1}, a_{2}\right)-\phi\left(b_{1}, b_{2}\right)<0$.

(i) Suppose that $k>0$. Consider two strictly positive arbitrary rational numbers $r_{1}$ and $r_{2}$ such that $r_{1}<k<r_{2}$. With $r_{1}=K_{1} / L_{1}, r_{2}=K_{2} / L_{2}, n_{1}=K_{1}+L_{1}$ and $n_{2}=K_{2}+L_{2}$, compared to the proof in part (a), (i), all inequalities before inequality (A4) switch sign, but (A4) remains true. One can further proceed as in (a), (i) to show that (A2) holds true.

(ii) Suppose that $k<0$. Consider two strictly negative arbitrary rational numbers $r_{1}$ and $r_{2}$ such that $r_{1}<k<r_{2}$. With $r_{1}=-K_{1} / L_{1}, r_{2}=-K_{2} / L_{2}, n_{1}=K_{1}+L_{1}$ and $n_{2}=K_{2}+L_{2}$, one can proceed as in part (a), (ii), paying heed to the signs of the first sets of inequalities, to show that (A2) holds true.

Returning to the proof of the theorem, put the right hand side equal to the number $R\left(\lambda_{1}, \lambda_{2}\right)$. Fixing $\left(b_{1}, b_{2}\right)$, the equation can be written as

$$
\begin{aligned}
& \phi\left(\lambda_{1} a_{1}, \lambda_{2} a_{2}\right)-\phi\left(\lambda_{1} b_{1}, \lambda_{2} b_{2}\right)=R\left(\lambda_{1}, \lambda_{2}\right)\left[\phi\left(a_{1}, a_{2}\right)-\phi\left(b_{1}, b_{2}\right)\right] \Leftrightarrow \\
& \quad \phi\left(\lambda_{1} a_{1}, \lambda_{2} a_{2}\right)=R\left(\lambda_{1}, \lambda_{2}\right) \phi\left(a_{1}, a_{2}\right)+\phi\left(\lambda_{1} b_{1}, \lambda_{2} b_{2}\right)-R\left(\lambda_{1}, \lambda_{2}\right) \phi\left(b_{1}, b_{2}\right) .
\end{aligned}
$$


With $\left(b_{1}, b_{2}\right)$ fixed, put $Q\left(\lambda_{1}, \lambda_{2}\right)=\phi\left(\lambda_{1} b_{1}, \lambda_{2} b_{2}\right)-R\left(\lambda_{1}, \lambda_{2}\right) \phi\left(b_{1}, b_{2}\right)$ and we get $\phi\left(\lambda_{1} a_{1}, \lambda_{2} a_{2}\right)=R\left(\lambda_{1}, \lambda_{2}\right) \phi\left(a_{1}, a_{2}\right)+Q\left(\lambda_{1}, \lambda_{2}\right)$. The solutions to this functional equation are, see Aczél et al. (1986):

$$
\phi\left(a_{1}, a_{2}\right)=a+b a_{1}^{-r_{1}} a_{2}^{r_{2}} \text { and } \phi\left(a_{1}, a_{2}\right)=a-r_{1} \ln \left(a_{1}\right)+r_{2} \ln \left(a_{2}\right),
$$

where $b>0, r_{1}$ and $r_{2}>0$, to incorporate USM. EM eliminates the logarithmic measure. The values of $a$ and $b$ do not matter since $F($.) is increasing. SI implies that $r_{1}=r_{2}$, such that we can write the mobility index as in the theorem. To see that

DISM (IDSM) requires that $r>(<)$, observe that the second derivative of $\left(\frac{y_{2 i}}{y_{1 i}}\right)^{r}$ with respect to $y_{2 i}$ is equal to

$$
r(r-1)\left(\frac{y_{2 i}}{y_{1 i}}\right)^{r-2}>(<) 0 \text { if and only if } r>(<) 1 .
$$

We still need to prove the independence of the axioms. As usual, we do this by proposing mobility measures that satisfy all axioms used, except one. The second column of the following table formulates measures that satisfy all axioms of the theorem, except the axiom indicated in the first column.

\section{AXIOM}

RISC.1 [Anonymity]

RISC.2 [Rep. Invar.]

RISC.3 [Subgr. Con.]

EM

USM

RSI

SI

\section{MEASURE}

$\frac{1}{n} \sum_{i=1}^{n}\left(\frac{y_{2 i}}{y_{1 i}}\right)^{r_{i}}$ with all $r_{i}>0$

$r_{i}=1$ if $\left(\frac{y_{2 i}}{y_{1 i}}\right)=\left(\frac{y_{21}}{y_{11}}\right)$ and $r_{i} \neq 1$ otherwise.

$\sum_{i=1}^{n}\left(\frac{y_{2 i}}{y_{1 i}}\right)^{r}$ with $r>0$.

$\frac{1}{n} \sum_{i=1}^{n}\left(\frac{y_{2 i}}{\frac{1}{n} \sum_{j=1}^{n} y_{1 j}}\right)^{r}$ with $r>0$.

$\frac{1}{n} \sum_{i=1}^{n}\left(\ln \left(y_{2 i}\right)-\ln \left(y_{1 i}\right)\right)$.

$\frac{1}{n} \sum_{i=1}^{n}\left(\frac{y_{1 i}}{y_{2 i}}\right)^{r}$ with $r>0$.

$\frac{1}{n} \sum_{i=1}^{n} \exp \left(\frac{y_{2 i}}{y_{1 i}}\right)$.

$\frac{1}{n} \sum_{i=1}^{n}\left(y_{2 i}\right)^{n_{2}}\left(y_{1 i}\right)^{-\eta_{1}}$ with $r_{1}, r_{2}>0$. 
Proof of Theorem 2. Perform a transformation of all variables by putting $\hat{y}_{1 i}=\exp \left[y_{1 i}\right]$ and $\hat{y}_{2 i}=\exp \left[y_{2 i}\right]$. We are now in the positive domain, and TSI and AI reduce to RSI and SI. Hence we can apply Theorem 1 in terms of the transformed variables. Returning to the original variables, and putting $c=r$, completes the proof of the theorem.

\section{REFERENCES}

Aczél, J., Lecture Notes on Functional Equations and their Applications, Academic Press, New York, 1966.

Aczél, J., S. Roberts, and Z. Rosenbaum, "On Scientific Laws without Dimensional Constants," Journal of Mathematical Analysis and Applications, 199, 389-416, 1986.

Atkinson, A. B., "The Measurement of Economic Mobility," in P. Eijgelshoven and L. van Gemerden (eds), Inkomensverdeling en Openbare Financiën, Opstellen Voor Jan Pen, Het Spectrum, Utrecht, 9-24, 1981.

Bernasconi, M. and V. Dardanoni, "Measuring and Evaluating Intergenerational Mobility: Evidence from Students Questionnaires," in U. Schmidt, and S. Traub (eds), Advances in Public Economics: Utility, Choice and Welfare: A Festschrift for Christian Seidl, Springer Verlag, Dordrecht, 173-96, 2005.

Boudon, R., Mathematical Structures of Social Mobility, Elsevier Scientific Publishing Company, Amsterdam, 1973.

Burkhauser, R. V. and J. G. Poupore, "A Cross-National Comparison of Permanent Inequality in the United States and Germany," Review of Economics and Statistics, 79, 10-17, 1997.

Burkhauser, R., D. Hotz-Eakin, and S. Rhody, "Labor Earnings, Mobility and Equality in the United States and Germany during the Growth Years of the 1980s," International Economic Review, 38, 775-94, 1997.

Chakravarty, S. R., B. Dutta, and J. A. Weymark, "Ethical Indices of Income Mobility,” Social Choice and Welfare, 2, 1-21, 1985.

Cowell, F. A., "The Measurement of Distributional Change: An Axiomatic Approach," Review of Economic Studies, 52, 135-51, 1985.

D'Agostino, M. and V. Dardanoni, "What's so Special about Euclidean Distance?" Social Choice and Welfare, 33, 211-33, 2009.

Dardanoni, V., "Measuring Social Mobility," Journal of Economic Theory, 61, 372-94, 1993.

Fields, G. S., "Income Mobility," Cornell University, I.L.R School (http:// digitalcommons.ilr.cornell.edu/workingpapers/19/) 2007.

Fields, G. S. and E. A. Ok, "The Meaning and Measurement of Income Mobility," Journal of Economic Theory, 71, 349-77, 1996.

_ - "Measuring Movement of Incomes," Economica, 66, 455-71, 1999.

Foster, J. and A. Sen, "On Economic Inequality after a Quarter Century," in A. Sen (ed.), On Economic Inequality, Oxford University Press, Oxford, 107-219, 1997.

Foster, J. and A. Shorrocks, "Subgroup Consistent Poverty Indices," Econometrica, 59, 687-709, 1991.

Gorman, W. M., "The Structure of Utility Functions," Review of Economic Studies, 35, 367-90, 1968.

Gottschalk, P. and E. Spolaore, "On the Evaluation of Economic Mobility," Review of Economic Studies, 69, 191-208, 2002.

Jenkins, S. and P. Van Kerm, "Trends in Income Inequality, Pro-Poor Income Growth, and Income Mobility," Oxford Economic Papers, 58, 531-48, 2006.

Maasoumi, E. and M. Trede, "Comparing Income Mobility in Germany and the U.S. using Generalized Entropy Mobility Measures," Review of Economics and Statistics, 83, 2001.

Markandya, A., "Intergenerational Exchange Mobility and Economic Welfare," European Economic Review, 17, 307-24, 1982.

Mitra, T. and E. A. Ok, "The Measurement of Income Mobility: A Partial Ordering Approach," Economic Theory, 12, 77-102, 1998.

Ruiz-Castillo, J., "The Measurement of Structural and Exchange Mobility," Journal of Economic Inequality, 2, 219-28, 2004.

Schluter, C., "Statistical Inference with Mobility Indices," Economics Letters, 59, 157-62, 1998.

Schluter, C. and M. Trede, "Local versus Global Assessment of Mobility," International Economic Review, 44, 1313-35, 2003.

Shorrocks, A. F., "Income Inequality and Income Mobility," Journal of Economic Theory, 19, 376-93, 1978.

Review of Income and Wealth (C) International Association for Research in Income and Wealth 2010 
Review of Income and Wealth, Series 57, Number 1, March 2011

Tsui, K.-Y., "Multidimensional Generalizations of the Relative and Absolute Inequality Indices: The Atkinson-Kolm-Sen Approach," Journal of Economic Theory, 67, 251-65, 1995.

Van Kerm, P., "What Lies Behind Income Mobility? Reranking and Distributional Change in Belgium, Western Germany and the USA," Economica, 71, 223-39, 2004.

Yasuda, S., "A Methodological Inquiry into Social Mobility," American Sociological Review, 29, 16-23, 1964. 